\title{
Effect of porous polycaprolactone beads on bone regeneration: preliminary in vitro and in vivo studies
}

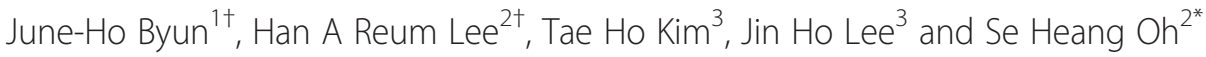

\begin{abstract}
Background: For the effective bone regeneration with appropriate pathological/physiological properties, a variety of bone fillers have been adapted as a therapeutic treatment. However, the development of ideal bone fillers is still remained as a big challenge in clinical practice. The main aims of this study are i) fabrication of a highly porous $\mathrm{PCL}$ beads; and ii) the estimation of the potential use of the porous PCL beads as a bone filler through preliminary animal study.

Results: The porous PCL beads with size range of $53 \sim 600 \mu \mathrm{m}(425 \sim 500 \mu \mathrm{m}$ dominantly) are fabricated by a spray/precipitation method using a double nozzle spray and PCL solution (in tetraglycol). The PCL beads show highly porous inner pore structure and the pores are interconnected with outer surface pores. For the preliminary animal study, we recognize that the porous PCL bead can induce the new bone formation from the outer surface of bone defect toward the bone marrow cavity through the bead matrix.
\end{abstract}

Conclusions: From the preliminary results, we can suggest that the highly porous $\mathrm{PCL}$ beads may be a promising candidate as a bone filler (scaffolding matrix) for the effective bone regeneration.

Keywords: Bone filler, Femur, Bone defect, Polycaprolactone (PCL), Porous bead

\section{Background}

Although an injury bone can be reconstructed spontaneously, large bone defects created by trauma, tumor resection, corrective osteotomy, and congenital deformity are considered as a notable challenge for orthopedic and oral/maxiallofacial surgeons [1]. For the effective bone regeneration with appropriate pathological/physiological properties, a variety of bone grafts including biological and synthetic biomaterials have been utilized as a therapeutic treatment. The biological grafts (autograft and allograft) are commonly used as a first line therapy for large-sized bone defect, but insufficient donor materials, inevitable donor site morbidity, risk of infection (autograft); and risk of immune response/disease transmission (allograft) remain as significant limitations in the clinical

\footnotetext{
*Correspondence: seheangoh@dankook.ac.kr

${ }^{\dagger}$ Equal contributors

2Department of Nanobiomedical Science \& BK21 PLUS NBM Global Research Center for Regenerative Medicine, Dankook University, Cheonan 330-714, Korea

Full list of author information is available at the end of the article
}

practice [2-4]. To solve the limitations, ceramic-based materials with similar mineral constituent of bone, such as hydroxyapatite (HA) and tri-calcium phosphate (TCP) have been utilized for the effective bone regeneration due to their biocompatibility, non-immunogenecity, osteoconductivity, bonding affinity with host bone, etc. [4-8]. However, their low reliability (i.e., weak mechanical strengths and high fragile failure rate) in wet environment which leads to difficulty for load-bearing applications and longterm degradation rate which can prohibit new bone growth into the defect site are considered as a limitation for clinical applications $[5,9,10]$. Recently, US Food and Drug Administration (FDA) approved biodegradable polymers [e.g., poly(glycolic acid) (PGA), poly(lactic acid) (PLA) and poly(lactic acid-co-glycolic acid) (PLGA), poly ( $\varepsilon$-caprolactone) $(\mathrm{PCL})$, polydioxanone (PDO)] with biocompatibility, predictable degradation rate and controllable mechanical properties are gained increasing interest as alternative matrices for bone regeneration [11]. Among them, the PCL is considered as a more promising matrix for bone regeneration compared to the other biodegradable 
polymers because of its no acidic by-products formation during degradation, flexibility (vs. PGA, PLA, PLGA); and relatively long-term structural stability which can provide a frame work during bone regeneration (vs. PGA, PLGA, PDO). Low et al. [12] demonstrated that the PCL matrix can allow biomimetic environment for the initial blood coagulation, cell infiltration, new blood vessel formation, and effective long-term osteogenesis. Moreover, Schantz et al. [13] reported that the PCL matrix is well tolerated in vivo and integrated with the host bone, suggesting that the PCL matrix may be a suitable graft for bone regeneration. Nevertheless the encouraging results, the use of PCL matrix as a bone filler is still limited, probably due to the concern about long-term remaining at applied site of dense PCL matrix which may prevent new bone formation. However, we expected that the highly porous PCL matrix may allow an appropriate environment for initial bone growth (by structural stability), accelerated degradation (by large surface area), sustained delivery of bioactive molecules (by high porosity), and thus become a good candidate as a bone filler.

Therefore, the main aims of this study are i) fabrication of a highly porous PCL bead; and ii) the estimation of the potential use of the porous PCL bead as a bone filler through preliminary animal study. To achieve this goal, porous PCL beads are fabricated by a spray/precipitation method using a double nozzle spray and PCL solution (in tetraglycol). The tetraglycol which is frequently utilized in parenteral delivery [14-16] is used as a nontoxic solvent for PCL. The preliminary animal study (femur defect rat model) to estimate the bone regeneration behavior by the porous PCL bead is also investigated.

\section{Method}

\section{Materials}

PCL (Mw 80,000 Da) and tetraglycol (glycofurol) as a nontoxic solvent for PCL were purchased from SigmaAldrich (USA). All other chemicals were analytical grade and were used as received. Ultrapure grade water $(>18$ $\mathrm{m} \Omega$ ) was purified using a Milli-Q purification system (Millipore Co., USA). For animal study, the porous PCL beads were sterilized by ethylene oxide (EO).

\section{Preparation of porous PCL beads}

Porous PCL beads were simply prepared by spray/ precipitation method using double nozzle spray. PCL pellets were dissolved in tetraglycol at $90^{\circ} \mathrm{C}$ (15 wt\%), and the PCL solution was immediately transferred in a $10 \mathrm{~mL}$ syringe. The warm solution was sprayed through a double nozzle spray with $\mathrm{N}_{2}$ purging of $2.5 \mathrm{~L} / \mathrm{min}$ (outer nozzle) into $50 \%$ ethanol solution (coagulation solution) to induce the solidification (precipitation) of PCL solution (Figure 1). Feeding rate of the solution was fixed to $60 \mathrm{~mL} / \mathrm{h}$ (inner nozzle, using syringe pump). The syringe and double nozzle spray were heated $\left(90^{\circ} \mathrm{C}\right)$ using a heating system equipped with heating tape (PID temperature controller, Model, TC130P; heating tape, Model, HT2510; Misung Scientific, Korea) to prevent precipitation of PCL during the process. The distance of tip-to-coagulation solution was $20 \mathrm{~cm}$. The precipitated PCL beads were maintained at coagulation solution for $6 \mathrm{hrs}$, then the PCL beads were washed out in excess water for $24 \mathrm{hrs}$ to remove residual tetraglycol and ethanol. The PCL beads were obtained by centrifugation and dried in a vacuum oven overnight, and the beads were separated in different size ranges $(53 \sim 100,100 \sim 200,200 \sim 300,300 \sim$ $425,425 \sim 500,500 \sim 600 \mu \mathrm{m})$ using standard testing sieves (Chunggye Industrial Co., Korea).

\section{Characterization of porous PCL beads Morphology observation and porosity measurement}

The morphology of prepared porous PCL beads was observed by a field emission scanning electron microscope (FE-SEM; Model S-4300, Hitachi, Japan). The crosssectional specimen was prepared by cutting them using a blade after being frozen in liquid nitrogen. The porosity of the PCL beads was estimated using mercury porosimetry (Poresizer 9320; Micromeritics, USA). To determine the porosity, it was assumed that the surface tension of mercury is 480 dyne/cm [17].

\section{Preliminary animal study}

Sprague-Dawley (SD) rats ( 250 g) were selected as an animal model to estimate the bone reconstruction potential by the use of porous PCL beads. The animal study was permitted from the Animal Care Committee of the Hannam University in Korea, and all surgical procedures were performed according to the guidelines. The rats were completely anesthesized with intramuscular injection of tiletamine/zolazepam (10 $\mathrm{mg} / \mathrm{kg}$; Zoletil $50^{\circ}$, Virbac Laboratories, France) and $2 \%$ xylazine hydrochloride $(2 \mathrm{mg} / \mathrm{kg}$;

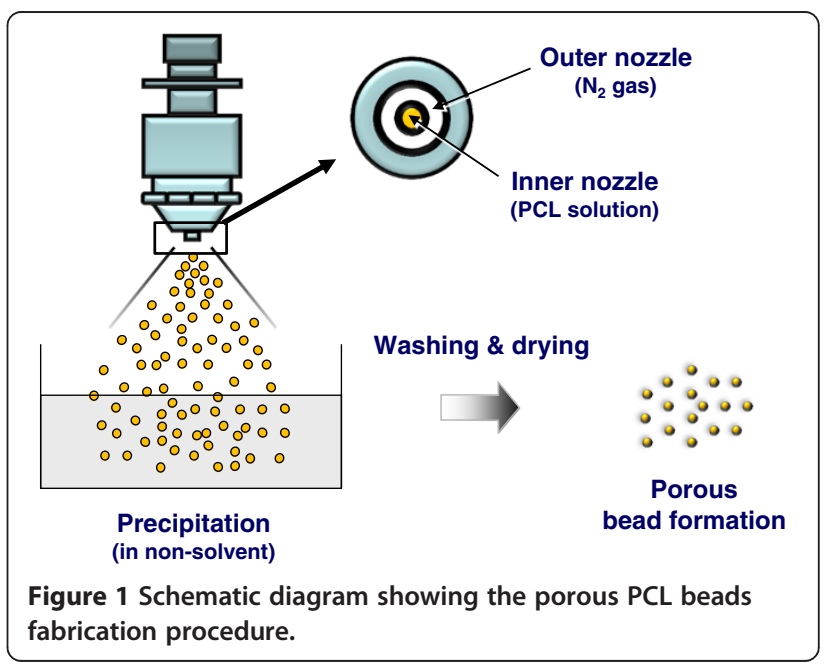


Rumpun ${ }^{\oplus}$, Byely, Korea), and placed in the prone position. The left leg was shaved and the skin surface discontaminated with $7 \%$ tincture of iodine. The front skin of the mid-femur in rats was incised straight and longitudinally at $30 \mathrm{~mm}$ in length. After splitting the muscle, the periosteum was stripped to expose the femoral bone surface. Two drill-holes (each hole, $\sim 4 \mathrm{~mm}$ in diameter) were created through the femoral cortex using a small tungsten carbide dental bur with a diameter of $0.8 \mathrm{~mm}$ in the anterior portion of the diaphysis of one femur $(10 \mathrm{~mm}$ position from the knee joint) (Figure 2). The bone defect was prepared with a very gentle surgical technique and continuous internal cooling with physiological saline solution. Then the porous PCL beads were implanted into the defect, and the wound was closed in two layers using 4-0 vicryl and 4-0 nylon sutures. The blank (w/o any treatment) was also studied as a control group. During surgery and 24 hrs later, all animals received subcutaneous injections of antibiotics (sulfadoxin and trimethoprin, 5:1, $15 \mathrm{mg} / \mathrm{kg}$ ) to minimize the risk of infections. At 4 weeks after implantation, the rats were sacrificed by an overdose of $\mathrm{CO}_{2}$ gas. For histological observation, the bone defects with surrounding femoral bone were harvested, and the specimens were fixed with $4 \%$ formaldehyde and decalcified in $10 \%$ formic acid. After dehydration procedure of the fixed specimens in a graded series of alcohol, the specimens were embedded in paraffin and cut into $5 \mu \mathrm{m}$ transverse sections in the defects. The sections were stained with Hematoxyline \& eosin (H\&E) and Masson's trichrome (MT) for observation by light microscopy (Model BX50F4, Olympus). For radiographic evaluation, the tissue specimens harvested from the femur of rat were frozen, and placed on an automatic axially-moving turntable to scan using a micro-computed tomography ( $\mu$-CT) system (Skyscan 1176, Skyscan N.V., Belgium)).

\section{Results and discussion}

Preparation and characterization of porous PCL beads

Porous PCL beads were prepared to estimate its feasibility as an bone filler for the effective bone regeneration.
The PCL is the most commonly utilized biodegradable polyesters with biocompatibility and predictable degradation rate for medical applications [18]. Their degradation is understood as a metabolism via tricarboxylic acid (TCA) cycle [19]. The porous PCL beads were fabricated by a simple spray/precipitation method using double nozzle spray and PCL solution. The gross appearance of prepared porous PCL beads with different size ranges and their surface/cross-sectional morphologies observed by SEM were shown in Figures 3 and 4.

It was observed that the size range of the prepared porous PCL beads in our procedure [purging rate of $\mathrm{N}_{2}$, $2.5 \mathrm{~L} / \mathrm{min}$ (outer nozzle) and feeding rate of PCL solution, $60 \mathrm{~mL} / \mathrm{h}$ (inner nozzle)] is $53 \sim 600 \mu \mathrm{m}$ and the porous PCL beads with size range of $425 \sim 500 \mu \mathrm{m}$ is more dominant than other size ranges. Their size distribution also can be controlled by the purging rate of $\mathrm{N}_{2}$ and/or feeding rate of polymer solution [higher $\mathrm{N}_{2}$ purging rate, smaller size dominantly (lower volume ratio of polymer solution to $\mathrm{N}_{2}$ gas); higher PCL solution feeding rate, larger size dominantly (higher volume ratio of polymer solution to $\mathrm{N}_{2}$ gas); not shown data), as expected. The porous PCL beads exhibited highly porous inner pore structures and the pores are interconnected with surface pores. The formation of porous structure can be understood by phase separation between polymer (PCL) solution and nonsolvent [solvent (tetraglycol)-nonsolvent (50\% ethanol) exchange] during the precipitation of the PCL solution in coagulation bath [20]. From the morphology results, we could expect that the highly porous structure can provide large surface area of PCL matrix and thus may accelerate the degradation rate which can allow appropriate space for new bone formation, moreover the porous beads may be a reservoir of bioactive molecules for bone regeneration (e.g., drugs, growth factors, cytokines, etc.). Therefore, we believed that the porous PCL beads may be a promising matrix for effective bone regeneration. The porosity of the porous PCL beads measured using mercury porosimetry were over $80 \%$, regardless of bead size. The porous PCL beads with
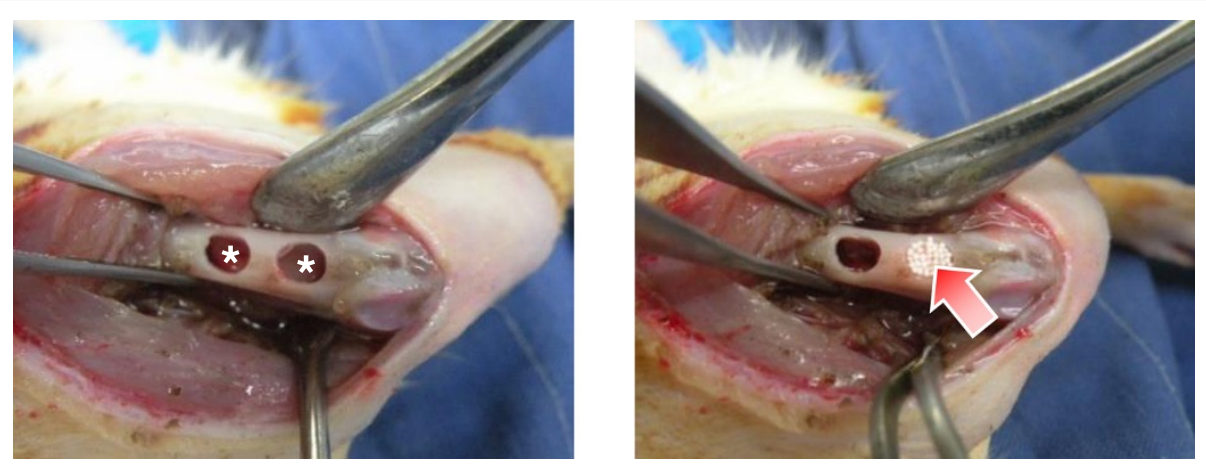

Figure 2 Photographs showing the implantation of porous PCL beads in a femoral defect of rat (*, bone defect; arrow, porous PCL beads). 


\section{(A)}
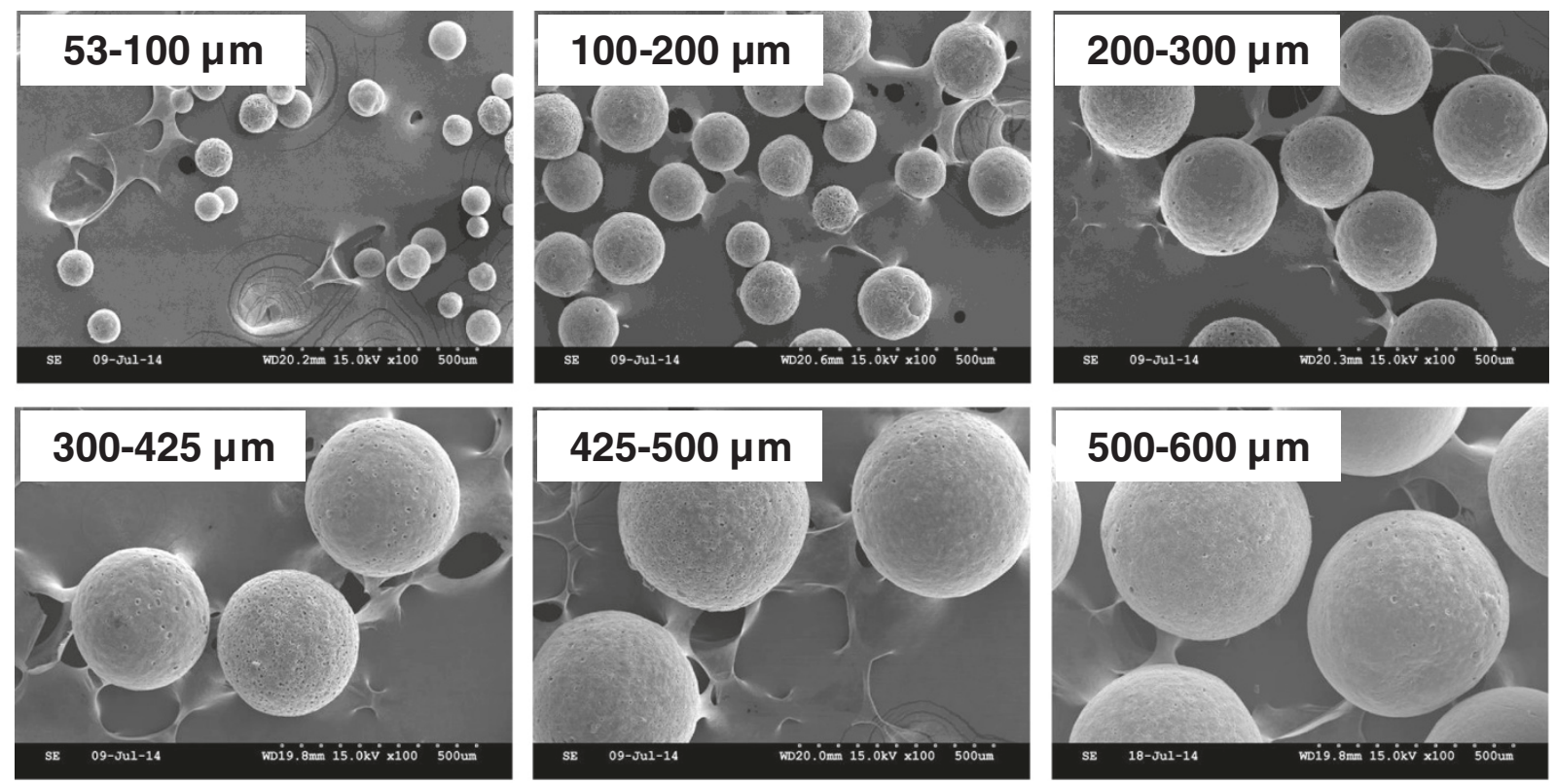

(B)

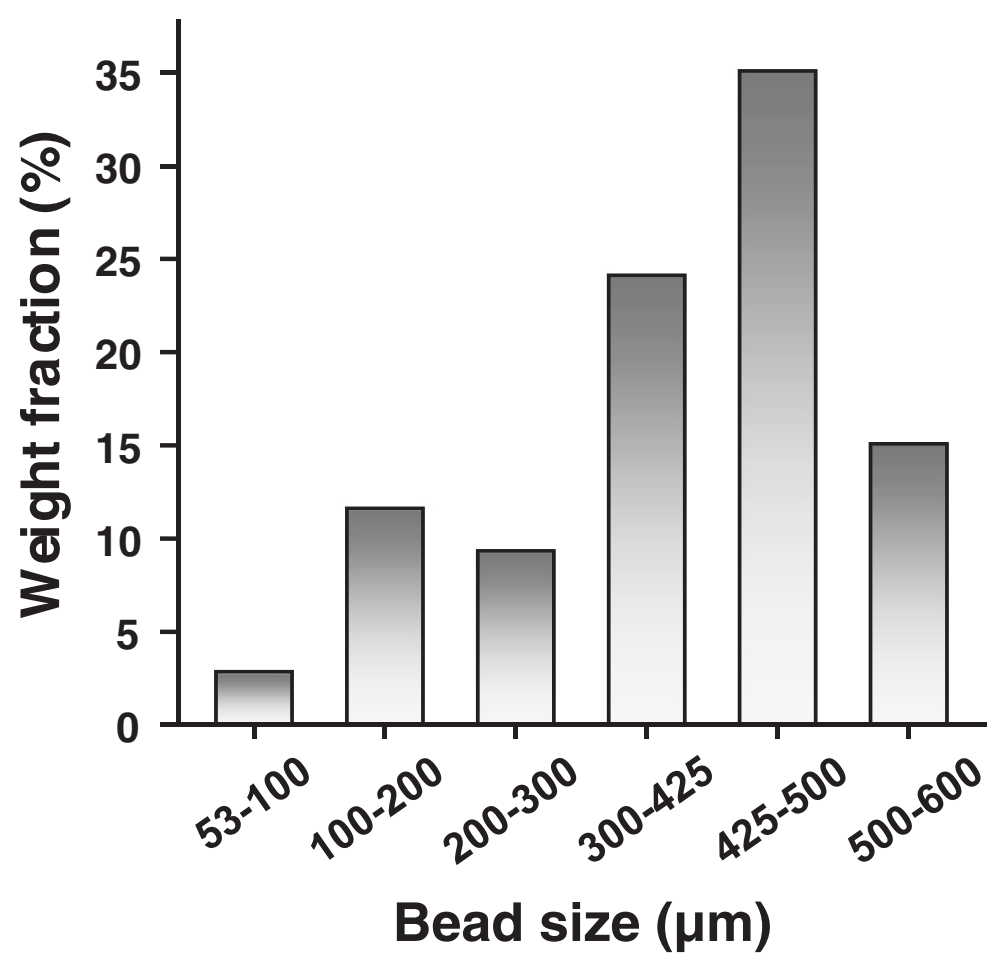

Figure 3 (A) SEM Photographs of the prepared porous PCL beads with different size range $(x 100)$ and $(B)$ their distribution expressed by weight fraction. 

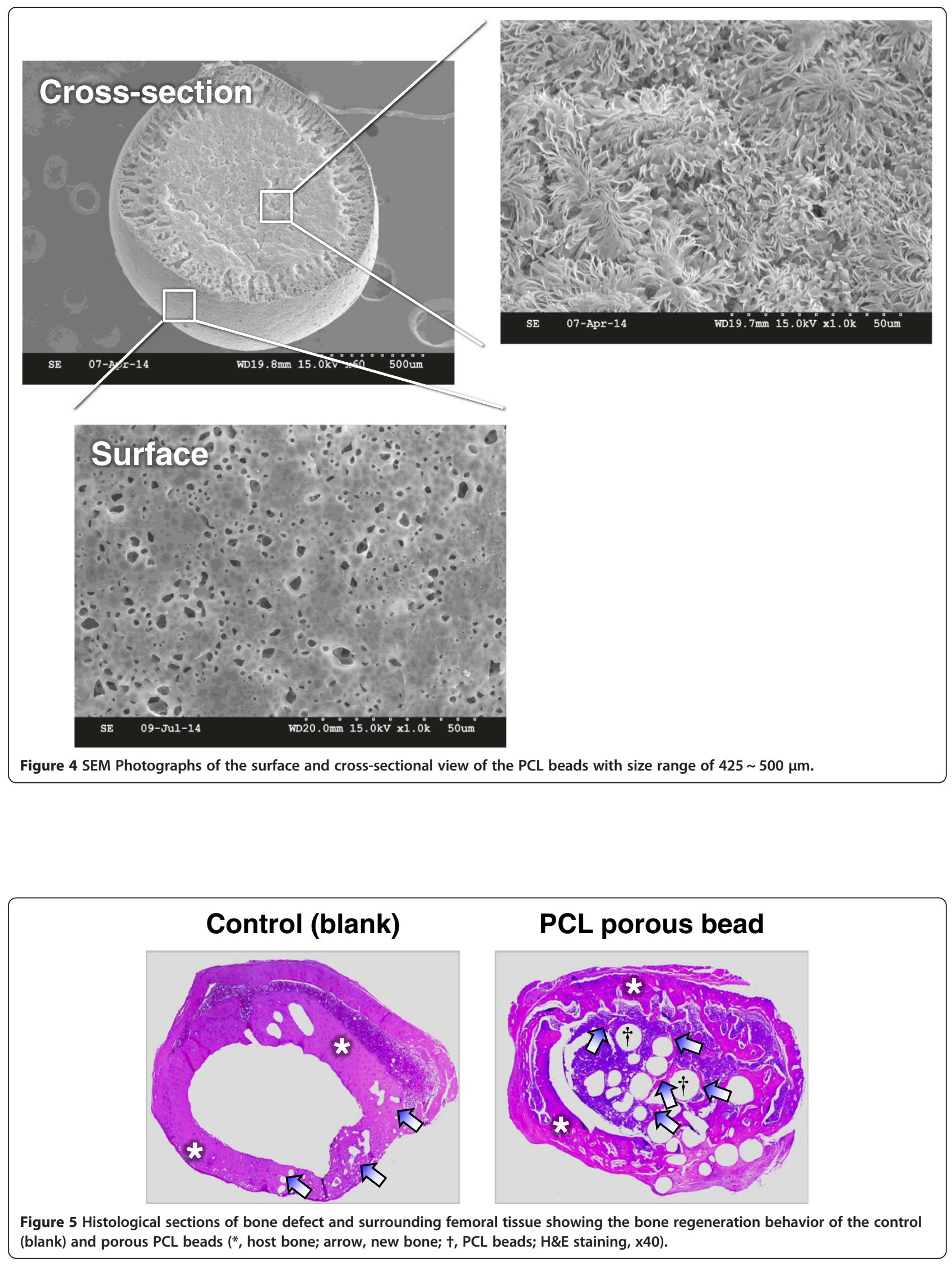


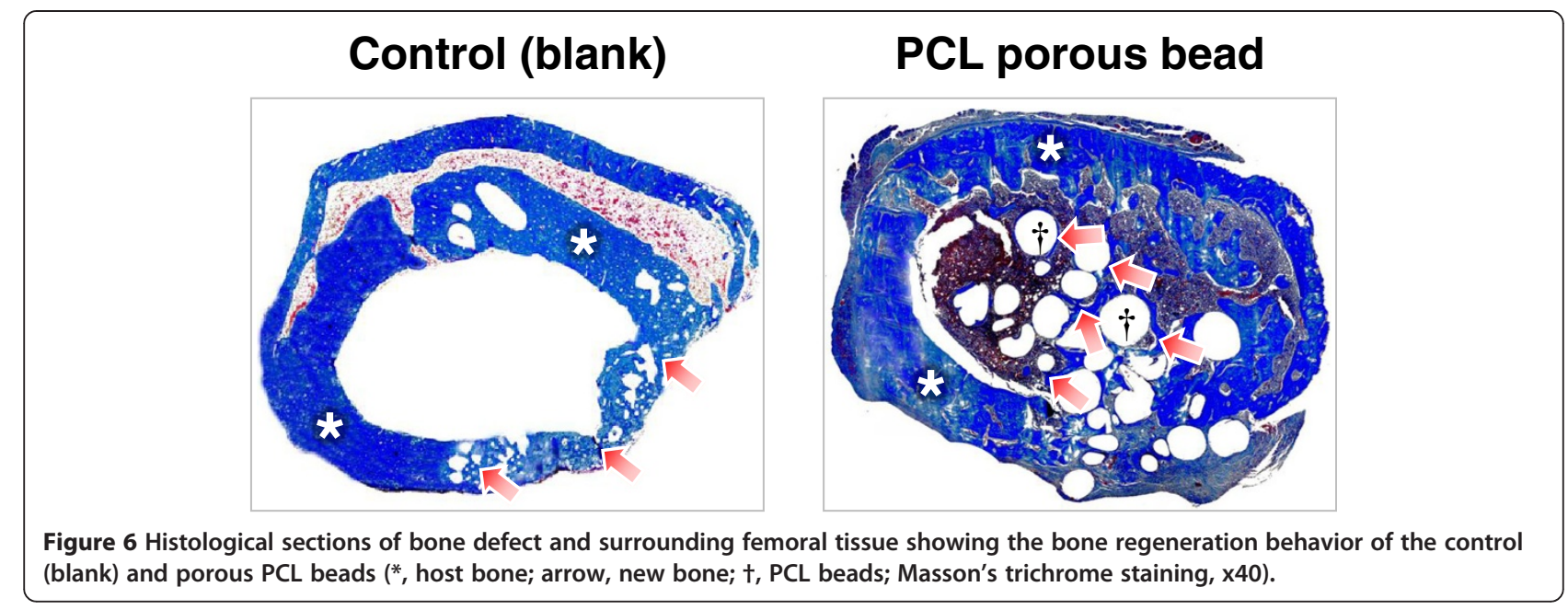

size range of $425 \sim 500 \mu \mathrm{m}$ were selected for the preliminary animal study using rat model [21].

\section{Preliminary animal study}

A SD rat model was used to estimate the bone regeneration behavior of the porous PCL beads. The rat femoral bone defect was chosen as the orthotopic model for this experiment. Bone defects of $\sim 4 \mathrm{~mm}$ diameter were created on a femur using a small tungsten carbide dental bur, and the porous PCL beads were filled into the defect. The blank (w/o any treatment) was also studied as a control group. At implantation, the porous PCL beads

\section{Longitudinal cross-section}
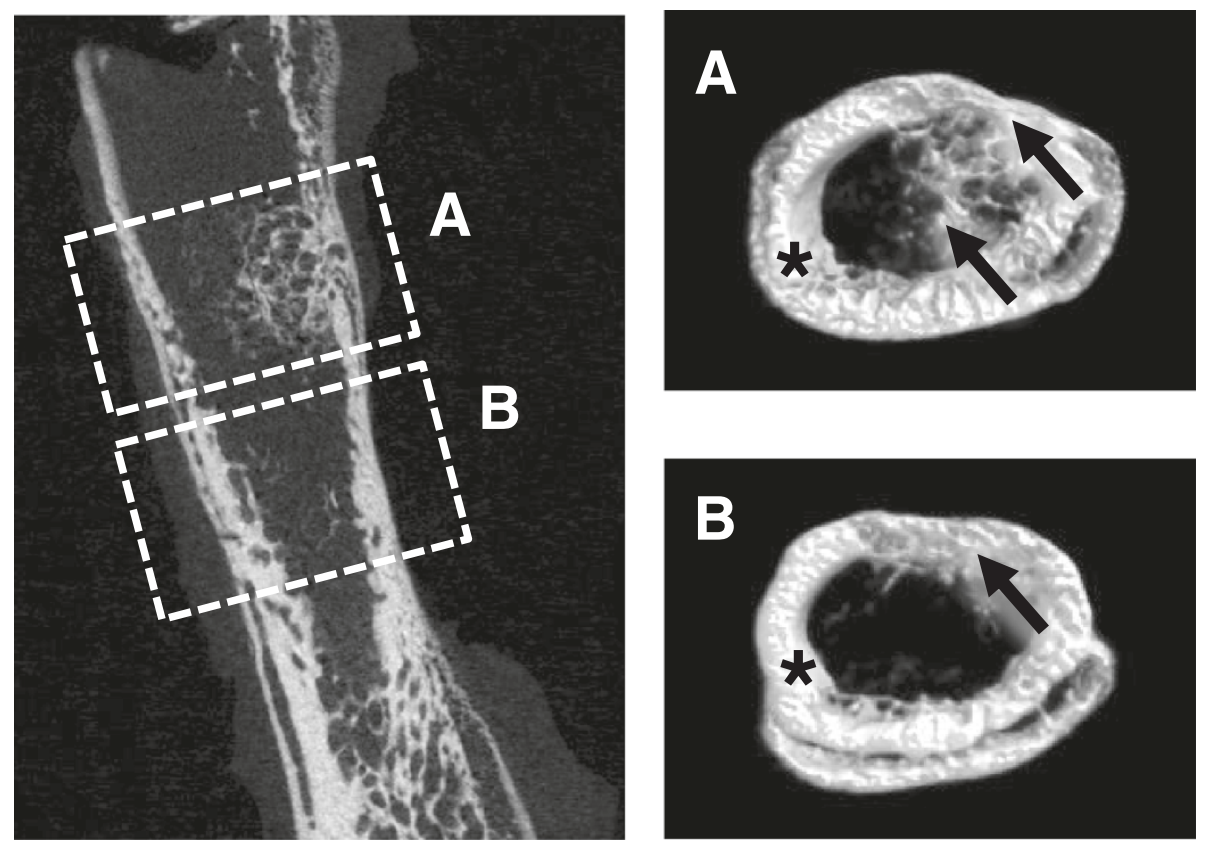

\section{A: Porous PCL bead; B: Control (blank)}

Figure $7 \mu$-CT images of femoral bone defect showing the mineralized bone regeneration behavior of (A) porous PCL beads and (B) control (blank) [*, host bone; arrow, regenerated (mineralized) bone]. 
were easily applied and formed well-packed structure in the defect site without floating by bleeding. During the breeding, all animals remained in sound health and did not show any wound complications. Figures 5 and 6 show the histological sections (after H\&E and MT staining) to compare bone reconstruction behavior in the bone defect filled with and without the porous PCL beads at 4 weeks after surgery. In the PCL bead group, the new bone was regenerated from the outer surface of bone defect toward the bone marrow cavity through the porous PCL beads. However, in the control (blank) group, the new bone was only reconstructed at outer surface of bone defect and the new bone formation in the marrow cavity was not detected. This indicates that the porous PCL bead itself can provide an appropriate environment for ingrowth of a variety of cells related with bone formation, and thus allow bone regeneration in the PCL bead matrix. The regenerated bone was grown along the porous matrix consisted of the PCL beads, suggesting that the beads are stably kept their porous structure without degradation during the bone regeneration and act as a scaffolding matrix which can allow the adhesion of bone-related cells and thus improve osteogenesis [22-24]. At 4 weeks after implantation, the mineralized bone regeneration was also studied using $\mu$-CT. The growth of mineralized bone tissues begun at the outer surface of all bone defects (Figure 7), and the mineralized bone tissue was infiltrated through the PCL bead matrix. This observation was consistent with the result of the histological results, showing that the porous PCL beads can effectively induce the bone regeneration. On the basis of our findings, we can suggest that the highly porous PCL beads fabricated by simple spray/precipitation method may be a candidate as a bone filler for the effective bone regeneration.

\section{Conclusions}

We prepared porous PCL beads by a spray/precipitation method using a double nozzle spray and PCL solution (in tetraglycol). It was observed that the size range of prepared porous PCL beads (purging rate of $\mathrm{N}_{2}, 2.5 \mathrm{~L} / \mathrm{min}$ and feeding rate of PCL solution, $60 \mathrm{~mL} / \mathrm{hr}$ ) is $53 \sim$ $600 \mu \mathrm{m}$ (dominant size range, $425 \sim 500 \mu \mathrm{m}$ ) and their size distribution can be controlled by the purging rate of $\mathrm{N}_{2}$ and/or feeding rate of polymer solution. The porous PCL beads showed highly porous inner pore structure and the pores are interconnected with outer surface pores. For the preliminary animal study, we recognized that the porous PCL bead can induce the new bone formation from the outer surface of bone defect toward the bone marrow cavity through the bead matrix. From the preliminary results, we could suggest that the highly porous PCL beads may be a promising candidate as a matrix for the bone regeneration. The long-term studies (i.e., in vivo degradation rate of the porous PCL beads and bone regeneration/ maturation behaviors through the matrix) using a large animal (porcine) to confirm the potential use of the porous PCL beads as a clinically applicable bone filler are in progress.

\section{Availability of supporting data}

The data sets supporting the results of this article are included within the article.

\section{Competing interests}

The authors declare that they have no competing interests.

\section{Authors' contributions}

Conceived and designed the experiments: J-HB, JHL, SHO. Performed the experiments: J-HB, HARL, SHO. Analyzed the data: J-HB, JHL, SHO. Wrote the paper: J-HB, SHO. All authors read and approved the final manuscript.

\section{Acknowledgement}

This research was supported by a grant of the Korea Health Technology R\&D Project through the Korea Health Industry Development Institute (KHIDI), funded by the Ministry of Health \& Welfare, Republic of Korea (grant number: HI13(1596)

\section{Author details}

${ }^{1}$ Department of Oral and Maxillofacial Surgery, Institute of Health Sciences, Gyeongsang National University School of Medicine, Jinju 660-702, Korea. ${ }^{2}$ Department of Nanobiomedical Science \& BK21 PLUS NBM Global Research Center for Regenerative Medicine, Dankook University, Cheonan 330-714, Korea. ${ }^{3}$ Department of Advanced Materials, Hannam University, Daejeon 305-811, Korea.

Received: 18 July 2014 Accepted: 19 October 2014

Published: 24 November 2014

\section{References}

1. Yang F, Wang J, Hou J, Guo H, Liu C: Bone regeneration using cell-mediated responsive degradable PEG-based scaffolds incorporating with rhBMP-2. Biomaterials 2013, 34:1514-1528.

2. Damien C, Parsons R: Bone graft and bone graft substitutes: a review of current technology and applications. J Appl Biomat 1991, 2:187-208.

3. Gazdag AR, Lane JM, Glaser D, Forster RA: Alternatives to autogenous bone graft: efficacy and indications. J AM Acad Orthop Sur 1995, 3:1-8.

4. Nandi SK, Kundu B, Ghosh SK, De DK, Basu D: Efficacy of nanohydroxyapatite prepared by an aqueous solution combusting technique in healing bone defects of goat. J Vet Sci 2008, 9:183-191.

5. Hench LL: Bioceramics: From Concept to Clinic. J Am Ceram Soc 1991, 74:1487-1510

6. Daculsi G, Hartmann DJ, Heughebaert M, Hamel L, Le Nihouannen JC: In vivo cell interactions with calcium phosphate bioceramics. J Submicrosc Cytol Pathol 1988, 20:379-384.

7. Jarcho M: Calcium phosphate ceramics as hard tissue prosthetics. Clin Orthop Relat Res 1981, 157:259-278.

8. LeGeros RZ, Parsons JR, Daculsi G, Driessens F, Lee D, Liu ST, Metsger S, Peterson D, Walker M: Significance of the porosity and physical chemistry of calcium phosphate ceramics biodegradation-bioresorption. Ann NY Acad Sci 1988, 523:268-271.

9. Grundel RE, Chapman MW, Yee T, Moore DC: Autogeneic bone marrow and porous biphasic calcium phosphate ceramic for segmental bone defects in the canine ulna. Clin Orthop 1991, 266:244-258.

10. Petite $H$, Viateau V, Bensaïd B, Meunier A, de Pollak C, Bourguignon M, Oudina K, Sedel L, Guillemin G: Tissue-engineered bone regeneration. Nat Biotechnol 2000, 18:959-963.

11. Oh SH, Lee JH: Hydrophilization of synthetic biodegradable polymer scaffolds for improved cell/tissue compatibility. Biomed Mater 2013, 8:014101.

12. Low SW, Ng YJ, Yeo TT, Chou N: Use of Osteoplug ${ }^{\mathrm{TM}}$ polycaprolactone implants as novel burr-hole covers. Singapore Med J 2009, 50:777-780. 
13. Schantz JT, Lim TC, Ning C, Teoh SH, Tan KC, Wang SC, Hutmacher DW: Cranioplasty after trephination using a novel biodegradable burr hole cover: technical case report. Neurosurgery 2006, 58(1 Suppl):ONS-E176.

14. Eliaz RE, Kost J: Characterization of a polymeric PLGA-injectable implant delivery system for the controlled release of proteins. J Biomed Mater Res 2000, 50:388-396.

15. Oh SH, Lee JY, Ghil SH, Lee SS, Yuk SH, Lee JH: PCL microparticle-dispersed PLGA solution as a potential injectable urethral bulking agent. Biomaterials 2006, 27:1936-1944.

16. Choi SJ, Oh SH, Kim IG, Chun SY, Lee JY, Lee JH: Functional recovery of urethra by plasmid DNA-loaded injectable agent for the treatment of urinary incontinence. Biomaterials 2013, 34:4766-4776.

17. Ritter HL, Drake LC: Pore-size distribution in porous materials. I. Pressure porosimeter and determination of complete macropore-size distribution. Ind Eng Chem 1945, 17:782-786.

18. Lin WJ, Flanagan DR, Linhardt RJ: A novel fabrication of poly(ecaprolactone) microspheres from blend of poly(e-caprolactone) and poly(ethylene glycol)s. Polymer 1999, 40:1731-1735.

19. Woodward SC, Brewer PS, Moatamed F: The intracellular degradation of poly(e-caprolactone). J Biomed Mater Res 1985, 44:437-444.

20. Oh SH, Kim JR, Kwon GB, Namgung U, Song KS, Lee JH: Effect of surface pore structure of nerve guide conduit on peripheral nerve regeneration. Tissue Eng Part C 2013, 19:233-243.

21. Kim TH, Oh SH, Chun SY, Lee JH: Bone morphogenetic proteins-immobilized polydioxanone porous particles as an artificial bone graft. J Biomed Mater Res Part A 2014, 102A:1264-1274.

22. Taguchi Y, Amizuka N, Nakadate M, Ohnishi H, Fujii N, Oda K, Nomura S, Maeda T: A histological evaluation for guided bone regeneration induced by a collagenous membrane. Biomaterials 2005, 26:6158-6166.

23. Oh SH, Kang SG, Kim ES, Cho SH, Lee JH: Fabrication and characterization of hydrophilic poly(lactic-co-glycolic acid)/poly(vinyl alcohol) blend cell scaffolds by melt-molding particulate-leaching method. Biomaterials 2003, 24:4011-4021.

24. Kellomäki $M$, Niiranen $H$, Puumanen $K$, Ashammakhi $N$, Waris T, Törmälä P: Bioabsorbable scaffolds for guided bone regeneration and generation. Biomaterials 2000, 21:2495-2505.

doi:10.1186/2055-7124-18-18

Cite this article as: Byun et al:: Effect of porous polycaprolactone beads on bone regeneration: preliminary in vitro and in vivo studies.

Biomaterials Research 2014 18:18.

\section{Submit your next manuscript to BioMed Central and take full advantage of:}

- Convenient online submission

- Thorough peer review

- No space constraints or color figure charges

- Immediate publication on acceptance

- Inclusion in PubMed, CAS, Scopus and Google Scholar

- Research which is freely available for redistribution 